\title{
Manejo nutricional integrado: herramienta clave para la agricultura sostenible
}

\author{
Juan José Hernández-Terrón ${ }^{1}$ \\ Francisco Gutiérrez-Rodríguez ${ }^{2 \S}$ \\ Rodolfo Serrato-Cuevas ${ }^{2}$ \\ Andrés González-Huerta ${ }^{2}$ \\ Enrique García-Rodríguez ${ }^{1}$
}

${ }^{1}$ Posgrado en Ciencias Agropecuarias y Recursos Naturales-Universidad Autónoma del Estado de MéxicoCampus Universitario El Cerrillo. Piedras Blancas, Toluca, Estado de México. CP. 50200. (htjuanjo@gmail.com; egarciar0105@hotmail.com). ${ }^{2}$ Centro de Investigación y Estudios Avanzados en Fitomejoramiento-Campus Universitario El Cerrillo. Piedras Blancas. Toluca, Estado de México. CP. 50200. (rserratoc@uaemex.mx; agonzalezh@uaemex.mx).

${ }^{\S}$ Autor para correspondencia: fgrfca@hotmail.com.

\section{Resumen}

El principal reto que enfrenta la agricultura es satisfacer la creciente demanda mundial de alimentos y al mismo tiempo reducir el impacto agrícola ambiental negativo. La nutrición de los cultivos depende principalmente de los fertilizantes minerales, lo cual es una amenaza para el medio ambiente y para la salud humana. Una alternativa para solventar esta problemática es el manejo nutricional integrado con fertilizantes minerales y abonos orgánicos para disminuir el uso de los fertilizantes minerales al tiempo que se favorece la productividad de los cultivos, así como la calidad de los productos agrícolas y del medio ambiente. La presente revisión recopila resultados de investigaciones enfocadas al manejo nutricional integrado, como parte del funcionamiento holístico de los agroecosistemas, en varios ámbitos: a) calidad del suelo; b) productividad y rentabilidad de los cultivos; c) calidad de los productos agrícolas; d) emisión de gases de efecto invernadero; y e) transferencia de nitrógeno al agua.

Palabras clave: agricultura sustentable, fertilización integrada, gases de efecto invernadero.

Recibido: julio de 2021

Aceptado: agosto de 2021 
La población mundial crecerá (en millones) de 7700 en 2019, a más de 9700 en 2050 (UNDESA, 2019). Dicha población en aumento requiere acceso permanente a alimentos inocuos, nutritivos y suficientes (FAO, 2019). Sin embargo, la agricultura basada en grandes cantidades de agroquímicos y de otros insumos externos, así como el consumo insostenible de alimentos, afectan al medio ambiente y a las sociedades debido a que propician afecciones a la salud humana y al tejido social (McKenzie y Williams, 2015). Por lo tanto, es necesaria una agricultura sostenible, la cual se define como la agricultura que garantiza la rentabilidad, la salud del medio ambiente y la equidad social y económica (FAO, 2015), lo cual se logra al armonizar los elementos de los agroecosistemas con su entorno (Gutiérrez et al., 2015).

Los fertilizantes minerales (FMs) tienen varias ventajas para su uso en la agricultura: solubilidad y proporciones de nutrientes altas y precisas, y precio relativamente bajo. Sin el uso de FMs la producción mundial de alimentos disminuiría en 50\% (Sarkadi et al., 2019), y se espera que el consumo mundial de N, P y K llegue a 199 Mt en 2023 (IFA, 2018). En suelos con bajos contenidos de $\mathrm{C}, \mathrm{N}$ y $\mathrm{P}$ disponible, la aplicación de dosis racionales de FMs, mejoran la calidad del suelo (Omari et al., 2017).

Sin embargo, los FMs tienen varios efectos nocivos, los cuales inician con su fabricación, al generar productos químicos tóxicos: gases y desechos que contaminan al suelo, cuerpos de agua y aire (Chandini et al., 2019). Además, entre el 60 y 90\% de la fertilización mineral (FM) aplicada se pierde (Bhardwaj et al., 2014) por ejemplo, parte del $\mathrm{N}$ se lixivia o se escurre como $\mathrm{NO}_{3}$, volatiliza como $\mathrm{NH}_{3}$ u óxidos de $\mathrm{N}$, lo que causa eutrofización de cuerpos de agua, efecto invernadero y lluvia ácida (Liu et al., 2014b). Incluso en condiciones ideales las plantas usan solo hasta 50\% del $\mathrm{N}$ aplicado, el resto se pierde de diversas formas, se estima que de 2 a $20 \%$ se volatiliza de 15 a $25 \%$ reacciona con los compuestos orgánicos del suelo y de 2 a $10 \%$ va hacia el agua superficial y subterránea (Chandini et al., 2019).

El empleo excesivo de FMs daña a los organismos que participan en la génesis y conservación del suelo, en la disponibilidad y reciclaje de nutrientes y el control de plagas y enfermedades de las plantas (Hernández et al., 2014) además causa enfermedades ambientales en humanos (Hernández et al., 2017) como la metahemoglobinemia (consumo de agua con alto contenido de nitratos) (Larios et al., 2015) asma, problemas cardiacos, etc. (lluvia ácida) (WHO, 2018). Los FMs no aportan al suelo los micronutrientes requeridos por las plantas, sino que suministran cadmio, arsénico y uranio, elementos tóxicos para la vida vegetal y animal (Sarkadi et al., 2019).

Por lo tanto, se deben evaluar los beneficios y perjuicios del uso de FMs, a fin de aplicarlos de forma adecuada a cada agroecosistema, considerando las fuentes, dosis, tiempo, lugar, eficiencia de uso de nutrientes y evaluación de riesgos (Sarkadi et al., 2019). Por otra parte, la recuperación y conservación de suelos agrícolas requiere, entre otras prácticas, del reciclado de residuos de plantas, estiércol animal y lodos residuales (Thangarajan et al., 2013), transformados en abonos orgánicos (AOs) como compost (Cp) o vermicompost (Vc), biochar, etc.

Los AOs benefician las propiedades del suelo y los rendimientos (Masunga et al., 2016; El-Naggar et al., 2019), mejoran en el suelo la agregación, aireación, humedad, micro biota, carbono orgánico (CO) del suelo (COS), N, la masa, diversidad (DM) y actividad microbiana (AM), capacidad de intercambio catiónico (CIC) y aniónico, $\mathrm{pH}$, densidad aparente (DA), estabilidad y disponibilidad de nutrientes (Bhardwaj et al., 2014). Además, AOs como el Vc contienen vitaminas, hormonas y enzimas promotoras del crecimiento vegetal (Doan et al., 2015). 
Sin embargo, en cultivos con necesidades nutrimentales altas y puntuales, los AOs podrían no suministrar suficientes cantidades de nutrientes en el momento oportuno (Hernández et al., 2014). Como alternativa, la FAO (1998) propuso el manejo nutricional integrado (MNI), el cual consiste en la aplicación de FMs + AOs, también las legumbres, los residuos de cultivos, subproductos industriales y biofertilizantes son ingredientes comunes en el MNI, con el cual se mejora la productividad del suelo de manera sostenible (Das et al., 2014). Con MNI, se pueden sustituir parcialmente los FMs y elevar la eficiencia de AOs y FMs (García-Mendivil et al., 2014).

Por lo cual, el objetivo de este trabajo es proveer a los investigadores, ingenieros y productores agrícolas información de trabajos científicos en los que se probó el MNI, para mejorar los efectos del MNI sobre varias áreas: calidad del suelo, productividad de los cultivos, sanidad vegetal, calidad de los productos agrícolas, impacto ambiental y conveniencia económica. Los criterios que se siguieron para incluir los trabajos fueron los siguientes: uso combinado de AOs y FMs, medición de efectos sobre alguna de las seis áreas objeto de este estudio y antigüedad de publicación no mayor a 10 años. Los trabajos se ordenaron de acuerdo con el área en la que el MNI tuvo influencia y dentro de cada área, los trabajos se agruparon con base en las características específicas en las que influyó el MNI.

\section{Calidad agrícola del suelo y manejo nutricional integrado}

En la calidad agrícola del suelo (CS) se refleja su capacidad para sostener la productividad biológica y profundiza en los atributos edáficos que influyen en la interacción interacción del suelo con el ambiente, con la provisión de nutrientes, con la fotosíntesis y el crecimiento de los cultivos (Wilson y Sasal, 2017). En las últimas décadas, el concepto de CS evoluciona hacia el concepto de 'salud del suelo', en el cual se capturan los atributos ecológicos del suelo que tienen implicaciones más allá de su capacidad para producir un cultivo en particular (Bünemann et al., 2018).

Dentro de las propiedades físicas del suelo más importantes, están el tamaño de partícula, estabilidad de agregados (EA), densidad aparente (DA), estructura (Zornosa et al., 2015), textura, profundidad, conductividad hidráulica, capacidad de retención de agua (CRA) y porosidad (Navarrete et al., 2011; García et al., 2012). Las propiedades físicas indican cómo el suelo acepta, infiltra, retiene y proporciona agua a las plantas, factores que influyen en el crecimiento de raíces, emergencia de plántulas, movimiento de fauna en el perfil e intercambio de gases (Etchevers et al., 2009).

Los atributos químicos, como $\mathrm{CO}$ total y lábil, $\mathrm{N}$ total y mineralizable, $\mathrm{pH}$, conductividad eléctrica (CE), CIC, etc., afectan la disponibilidad y calidad del agua, a la disponibilidad de los nutrimentos para las plantas y microbios y a la capacidad amortiguadora del suelo (Navarrete et al., 2011; García et al., 2012). Cabe resaltar, que el COS está relacionado con la materia orgánica de suelo (MOS) y en el enfoque holístico y preventivo de los agroecosistemas sostenibles la MOS es fundamental (Gupta et al., 2017) debido a que es almacén y fuente de nutrientes, mejora las propiedades de los suelos, aporta microbios benéficos que ponen disponibles nutrientes, estimulan el crecimiento vegetal y suprimen patógenos (Hernández et al., 2014; Masunga et al., 2016). Las propiedades biológicas y bioquímicas del suelo como $\mathrm{C}$ y $\mathrm{N}$ de la biomasa microbiana (CBM y NBM), respiración basal (RB), actividades enzimáticas, DM, AM, etc., indican cambios tempranos en la MOS y en el reciclaje y dinámica de nutrientes (Navarrete et al., 2011; Fuentes et al., 2016). 
En varios cultivos con MNI se han reportado mejoras en la fertilidad del suelo, por ejemplo, en el sistema arroz (Oryza sativa), trigo (Triticum aestivum), con la aplicación FMs $+12 \mathrm{t} \mathrm{ha}^{-1}$ de Cp aumentó la disponibilidad de $\mathrm{N}, \mathrm{P}, \mathrm{K}, \mathrm{Ca}$ y $\mathrm{Mg}$, al tiempo que disminuyeron el pH y la adsorción de $\mathrm{Na}$ del suelo, lo cual se explica por la formación de ácidos, liberación de Ca y lixiviación de $\mathrm{Na}$ (Sarwar et al., 2008). Además de acrecentar el contenido de nutrientes, el MNI con residuos fúngicos + FMs amortiguó la acidificación del suelo causada por los FMs en el cultivo de arroz (Shi et al., 2019).

En el cultivo de soja (Glycine max) y trigo, la aplicación durante 21 años de FMs + estiércol de granja (EG) llevó a mejores niveles de micronutrientes extractables (Fe, $\mathrm{Mn}, \mathrm{Zn}$ y $\mathrm{Cu}$ ) comparado con la FM (Choudhary et al., 2018). Respecto a la fertilidad residual del suelo, en trigo con MNI ( $8 \mathrm{t}$ de $\mathrm{Cp} \mathrm{ha}^{-1}$ ), en los ensayos corrientes (año 1) y residuales (año 2), aumentó el P disponible 162 y $173 \%$, la MOS 108 y 104\%, el Ca intercambiable 16.7 y $17.4 \%$ y la CIC 15.4 y $17.1 \%$, respectivamente (Demelash et al., 2014). La fertilidad residual del suelo del cultivo de trigo se benefició con el MNI que incluyó el uso de 100\% FM + EG + Azotobacter + Zn + Mn + Fe (Singh et al., 2017).

El MNI de sistemas arroz-trigo y algodón (Gossypium hirsutum) trigo, con la inclusión de Cp de residuos municipales mejoró la DA y la resistencia a la penetración, y no hubo acumulación de metales pesados (zinc, cadmio, cromo, plomo y níquel) (Qazi et al., 2009). Con la inclusión de biochar en el MNI, además de mejorar la CS, se inmovilizan los metales pesados (Šimanský et al., 2019). El MNI también mejora los atributos biológicos del suelo. Liu et al. (2017) probaron en arrozales un índice que incluyó los nutrientes disponibles, CBM y actividad enzimática involucrada en los ciclos del C, N, P y S y encontraron que con el uso de FMs + estiércol de cerdo se obtuvo el mayor puntaje (0.85), comparado con el control (0.72) y con la FM sola (0.77), FM + abonos verdes (AVs) (0.81) y FM + paja (0.79).

En el cultivo de maíz (Zea mays), con FM + Vc se dieron las mayores mejoras en cuanto a CIC, disponibilidad de agua y $\mathrm{P}$, esto se debió a que el Vc estimuló la AM, la mineralización de nutrientes y la secreción de enzimas de las raíces (Doan et al., 2015). El MNI con bocashi, en el cultivo de maíz aumentó la colonización micorrízica (Álvarez-Solís et al., 2010) así como el CBM (Bautista-Cruz et al., 2015). Con el empleo de $\mathrm{Cp}+$ fertilizante bacteriano se incrementó la diversidad de comunidades bacterianas y fúngicas en el maíz (Zhen et al., 2014). Con MNI (50\% FM recomendada + $1250 \mathrm{~kg}$ de $\mathrm{MO} \mathrm{ha}^{-1}$ ) en banano (Musa paradisiaca) se elevaron la diversidad bacteriana, el pH y la actividad de la fosfatasa ácida, pero al aumentar la aplicación de FMs, dichos atributos del suelo disminuyeron (Sun et al., 2018).

Srivastava et al. (2012) reportaron que en plantaciones de cítricos el cambio gradual de FMs hacia AOs favoreció la DM. Con MNI en tomate (Lycopersicom esculentum) se incrementaron la MOS, el C lábil (Ren et al., 2014) y la RB (Hernández et al., 2014). Los estudios de MNI a largo plazo, muestran un efecto positivo sobre el COS y sobre las propiedades físicas del suelo. En ese sentido, Cai et al. (2019) aplicaron MNI en el sistema arroz-trigo y observaron que durante los primeros 10 años aumentó el COS y luego se estabilizó en los 15 años siguientes. En otro estudio, con la aplicación durante más de 40 años de FMs +15 t ha $^{-1}$ de EG en el sistema arroz-trigo se mantuvo el nivel de $\mathrm{CO}$ y se mejoraron algunas propiedades físicas del suelo, como son DA, conductividad hidráulica y CRA (Pant et al., 2018), pero disminuyó el contenido de zinc (Ram et al., 2016). 
En otro trabajo, el MNI con FMs + $5 \mathrm{t} \mathrm{ha}^{-1}$ de EG, después de 41 años de tratamiento aumentó el tamaño y estabilidad de los agregados del suelo (Tripathi et al., 2014). Mediante un meta-análisis elaborado con resultados de investigaciones realizadas de 1989 a 2016, se detectó que en el sistema arroz-trigo bajo MNI, el pH aumentó respecto al control (sin FMs ni AOs), en $1.15 \%$ en el suelo del cultivo de arroz, pero en trigo disminuyó $0.55 \%$, además el COS se elevó $23.2 \%$ y $16.2 \%$ respecto a la sola aplicación de FMs en arroz y trigo, respectivamente, el MNI también aumentó el NBM y el CBM. Se debe resaltar que los efectos del MNI fueron influenciados por el tipo de suelo, ya que las diferencias encontradas en el pH, NBM y CBM, fueron mayores en suelo francoarcilloso que en suelo arcilloso (Sharma et al., 2019).

\section{Manejo nutricional integrado y productividad de cultivos agrícolas}

Los beneficios que presenta el MNI sobre la CS ayudan a crear agrosistemas sostenibles (Cai et al., 2019), esto se maximiza en suelos poco productivos, ya que para la producción y aplicación de grandes cantidades de AOs se requeriría cubrir altos costos laborales y a veces la disponibilidad de materias primas es limitada (Wang et al., 2015). En el cultivo de maíz, con la aplicación de 50\% FM + AOs (composta, bocashi y humus de lombriz) se acrecentó el rendimiento en 3.8, 12.7 y $11.5 \%$ respecto al $100 \% \mathrm{FM}$, mientras que con $100 \% \mathrm{FM}+\mathrm{AOs}$, el incremento fue de 17.7, 21.9 y $30.5 \%$, respectivamente (Álvarez-Solís et al., 2010).

En otro estudio de MNI se evaluaron en plantas de maíz algunos parámetros que se relacionan con el rendimiento del cultivo, y los mayores valores de altura de planta, peso seco, índice de área foliar, fotosíntesis y conductancia estomática se registraron con la aplicación de EG + FMs (Efthimiadou et al., 2010). También la absorción de nutrientes (N, P, K, Zn, Fe y Mn) se elevó con el MNI (FMs + EG + bacterias solubilizadoras de P + Azotobacter + Zn + Fe + Mn) (Singh et al., 2017). En el cultivo de mostaza, el radio, índice y duración del área foliar, la tasa de asimilación neta (intercambio de $\mathrm{CO}_{2}$ por área foliar), clorofila, fotosíntesis y crecimiento del cultivo, fueron mayores con MNI (75\% FM + Vc a $2.5 \mathrm{t} \mathrm{ha}^{-1}$ ) comparados con 100\% FM (Mondal et al., 2017).

Por otro lado, en el cultivo de tomate con MNI (60\% FM + Cp, Vc o AVs) se obtuvieron rendimientos de frutos, altura y peso seco de plantas similares a los obtenidos con la FM al 100\% (Hernández et al., 2014; Ilupeju et al., 2015). Con la adición de biofertilizantes en el MNI las mejoras en los rendimientos son todavía mayores (Chatterjee et al., 2014; Verma et al., 2015); por ejemplo, el MNI con Cp inoculado con Trichoderma lleva a un rendimiento $43.8 \%$ mayor que con FM (Abedin et al., 2018). Se ha observado que la aplicación de gallinaza $+\mathrm{N}$ mineral en el cultivo de chile (Capsicum annuum) aumentó la temperatura del suelo, con lo cual se aceleraron las reacciones enzimáticas relacionadas con la maduración de los frutos (Macías et al., 2012).

Una evaluación de 25 años (1991 a 2015) con la aplicación de EG + FM, mostró que los rendimientos promedios de trigo y maíz (1.6 y $5.8 \mathrm{t} \mathrm{ha}^{-1}$ ) fueron mayores que con FM (0.97 y 2.65 $\left.\mathrm{t} \mathrm{ha}^{-1}\right)$, respectivamente. En otro estudio, con 30 años de MNI de soja y trigo, los rendimientos fueron mayores que con FM; sin embargo, aun con MNI bajaron los rendimientos a través del tiempo, debido a que el P, K y Zn disponibles disminuyeron (Bhattacharyya et al., 2008).

El MNI en cítricos mantuvo la productividad a largo plazo (Srivastava et al., 2012). Un metaanálisis del sistema arroz-trigo detectó el aumento del rendimiento con MNI, en 2.5, 29.2 y $90.9 \%$ sobre la FM, AOs y control, respectivamente (Sharma et al., 2019). Otro meta-análisis mostró que la FM + paja acrecienta el rendimiento de los cultivos 5.1\% más que la FM sola (Xia et al., 2018). 


\section{Manejo nutricional integrado y sanidad vegetal}

La calidad de la nutrición de las plantas determina sus características histológicas y morfológicas, así como su resistencia y capacidad para suprimir patógenos (Gupta et al., 2017), pero la aplicación excesiva y desbalanceada de FMs vuelve a las plantas más susceptibles a enfermedades (Wu et al., 2015). Por otro lado, una buena sanidad vegetal se favorece con la existencia en los agroecosistemas de competencia microbiana, hiperparasitismo, antibiosis, resistencia sistémica adquirida e inducida en los cultivos, inactivación de la proliferación de patógenos e influencia de las propiedades fisicoquímicas de los AOs (Mehta et al., 2014).

El MNI con balance de AOs y FMs ha demostrado buenas condiciones para la DM, mejora la nutrición vegetal, la resiliencia del ecosistema y la competencia contra patógenos (Sun et al., 2018). Sin embargo, dichos resultados no siempre se dan (Postma y Schilder, 2015), esto debido que la supresión de enfermedades depende de múltiples y complejas interacciones de los componentes del suelo de diferentes cultivos, prácticas de manejo, fertilizantes y ambientes (Sun et al., 2018).

Por ejemplo, en un ensayo de tizón tardío en tomate se registró menor incidencia (13.8\%) con 75\% $\mathrm{FM}+\mathrm{Vc}\left(4 \mathrm{t} \mathrm{ha}^{-1}\right)+$ biofertilizante en comparación con la FM sola (31.6\%) (Chatterjee y Khalko, 2013). En crisantemo, Pinto et al. (2013) Estudiaron el efecto del MNI con diversos AOs sobre la supresión de Fusarium y encontraron que la supresión fue mayor en la medida que se elevó la dosis de $\mathrm{Cp}$, pero el adicionar biofertilizante, hidrolizado de pescado, quitosano o Trichoderma, no tuvo efecto sobre la enfermedad, lo anterior sugiere que la supresión depende de la interacción de factores químicos y microbiológicos diversos.

En banano, el nivel de la supresión de Fusarium y de la CS tuvo correlación negativa $(\mathrm{r}=-0.92$, $p<0.01) ; 85.9 \%$ de la varianza fue explicada por suministro de $\mathrm{P}$ y K $(54.3 \%)$ y por la AM (31.6\%). El suministro de P y K favoreció a dosis altas de MO (500 o 750 g planta $^{-1}$ ) + FM al 100 o 50\%, mientras que la AM se incrementó con 25\% FM + MO. Con 50\% FM sin MO se estimuló la AM, pero con 100\% FM sin aplicación de MO, aumentó la incidencia de enfermedad (Sun et al., 2018).

\section{Manejo nutricional integrado y calidad de productos agrícolas}

El manejo nutricional afecta la calidad de los cultivos. Un estudio en trigo registro que con MNI, en las plantas se duplicó el contenido de N, Ca y Mg y se cuadruplicó el P (García-Medívil et al., 2014), además, en otro estudio se registró el aumento de la proteína en grano (Singh et al., 2017). En el cultivo de tomate, los AOs sin FM generaron menor cantidad de $\mathrm{N}$ en hojas y frutos, mientras que con MNI se obtuvieron frutos de buena calidad en cuanto a tamaño, firmeza, solidos solubles, acidez titulable, macro y micronutrientes (Hernández et al., 2014), en otras investigaciones con MNI de tomate también se incrementó el licopeno (35.5\%), la actividad antioxidante (de 24 a 63\%), las enzimas de defensa (de 11 a 54\%) y la vitamina C (Ilupeju et al., 2015; Verma et al., 2015).

En el cultivo de chile (Capsicum spp), la capsaicina y ácido ascórbico en frutos, aumentaron con la aplicación de $75 \% \mathrm{~N}$ de torta de neem $+25 \% \mathrm{~N}$ de urea, pero con $25 \% \mathrm{~N}$ de EG $+75 \%$ $\mathrm{N}$ de urea, disminuyeron los niveles de capsaicina (Pariari y Khan, 2013). En el cultivo de chile, la aplicación de Vc sólo o con FM incrementó la acidez, proteína, carotenos, licopenos y vitamina $\mathrm{C}$ en frutos, pero el Ca fue mayor con el control que con MNI (Das et al., 2016b; Premamali et al., 2019). 
El MNI de mostaza (Brassica campestris) elevó el contenido de clorofila, azúcar y prolina (Mondal et al., 2017), esto fue gracias a la mejora de la biosíntesis de clorofila y de la fotosíntesis, lo anterior se debió a que el MNI con Vc y Azotobacter aumentó la fijación y disponibilidad de N, la producción de sustancias promotoras del crecimiento vegetal y la absorción de nutrientes (Singh $e t$ al., 2014). En el cultivo de gladiolo (Gladiolus grandiflorus), el empleo de Vc + Cp inoculado con Trichoderma $+25 \%$ FM mejoró el tiempo de brote, período para plantación, número y peso de cormos, longitud de espigas y raquis, número de espigas y floretes (Akter et al., 2017).

Con el MNI en albahaca (Ocimum basilicum) se incrementó el rendimiento de aceite (metil chavicol y linalol), en comparación con los AOs o con la FM (Anwar et al., 2005) y en el cladodio de nopal (Opuntia ficus-indica) se elevó el contenido de zinc y boro, pero el de Mn fue mayor con la FM (Santiago-Lorenzo et al., 2016).

\section{Manejo nutricional integrado e influencia del $\mathrm{C}$ y $\mathrm{N}$ en el ambiente}

A nivel global las reservas de COS están agotadas en los agroecosistemas (Lal, 2018). La agricultura orgánica beneficia al ambiente y a la salud humana, pero podría exacerbar las emisiones de gases de efecto invernadero (GEI) a través de una mayor ocupación de terrenos para compensar la disminución de rendimientos (Smith et al., 2019). Por otra parte, la inadecuada aplicación agrícola de $\mathrm{N}$ ocasiona problemas ambientales y de salud (Liu et al., 2014b). Como alternativa a esta problemática, el MNI mejora la captura de C del suelo (CCS), mitiga las emisiones de GEI (Hua et al., 2014) y las pérdidas de $\mathrm{N}$ hacia el ambiente (Ren et al., 2014). Sin embargo, el C de residuos de cosecha sobre la superficie del suelo, estimula la AM, por lo que el COS se transforma en $\mathrm{CO}_{2} \mathrm{O} \mathrm{CH}_{4}$ a través de la respiración aeróbica y anaeróbica (Liu et al., 2014a).

En cambio, enterrar la paja incrementa la CCS y la retención de N, por lo que reduce las emisiones de GEI y las pérdidas de N (Yang et al., 2019). Lo anterior, evidencia la necesidad de

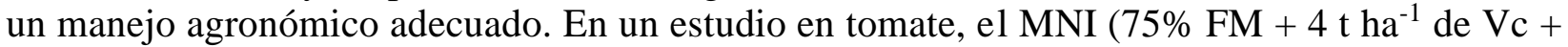
biofertilizante) mejoró la eficiencia de uso de $\mathrm{N}$ (Chatterjee et al., 2014). El MNI también disminuyó el agua de escorrentía y la lixiviación de $\mathrm{NH}_{4}{ }^{+}$y $\mathrm{NO}_{3}{ }^{-}$(Doan et al., 2015) además, favoreció la reproducción de microbios benéficos, el COS y la fijación y uso del N (Ojo et al., 2015). En el cultivo de albahaca, el CBM y NBM fueron más altos en suelos con MNI con Vc, comparados con los de la FM.

Esto se debe al efecto residual del Vc sobre la CCS, por la alta producción de biomasa, rizo deposición de materiales carbonosos y tejido desprendido (Anwar et al., 2005). En arrozales, el MNI con balance en las proporciones de FMs y AOs mejoró la CCS y la mineralización del C (MC), pero el uso excesivo de AOs o FMs produjo los efectos inversos (Shi et al., 2019). En rotaciones de trigo de invierno-maíz de verano, la FM ( $\mathrm{N}$ y P) aplicada por nueve años no alteró el C lábil, el microbiota del suelo, ni la MC; mientras que el uso de paja + FMs incrementó el C lábil, los microbios relacionados con los fosfolípidos y la MC (Li et al., 2018a).

En rotaciones trigo-soja y trigo-maíz bajo MNI durante nueve años, el $\mathrm{CO}$ y el $\mathrm{N}$ del suelo tuvieron aumentos de entre 14 y $37 \%$, esto gracias a que el $\mathrm{C}$ y $\mathrm{N}$ de los AOs se almacenan en fracciones recalcitrantes asociadas a los minerales del suelo (Li et al., 2018b). Con 46 años de MNI en el sistema maíz-trigo se incrementaron el CBM, RBS, MC y cociente microbiano, en comparación con la FM (Kaur et al., 2019). Un meta-análisis enfocado en el MNI con empleo de residuos de cultivos, detectó que con retorno de la paja, el COS aumentó $14.9 \%$ y la absorción de N por los cultivos creció $10.9 \%$, esto gracias a la inmovilización microbiana de N. 
En arrozales, el MNI aminoró las pérdidas de $\mathrm{N}_{2} \mathrm{O}(17.3 \%)$, la lixiviación de $\mathrm{N}(8.7 \%)$ y la escorrentía (25.6\%). Sin embargo, en cultivos no inundados, con el uso de FM + paja se intensificaron las emisiones de $\mathrm{N}_{2} \mathrm{O}(21.5 \%)$ y de $\mathrm{NH}_{3}(17 \%)$, esto debido al aumento de la nitrificación-desnitrificación y de la actividad de la ureasa del suelo. Las emisiones de $\mathrm{NH}_{3}$ y $\mathrm{N}_{2} \mathrm{O}$ disminuyeron a medida que la relación paja/C y el contenido de arcilla del suelo fueron mayores; pero a escala mundial, el retorno de la paja aumenta las pérdidas de $\mathrm{N}_{-} \mathrm{NH}_{3}$ (Xia et al., 2018).

En sistemas maíz-soja-trigo, dependiendo del manejo nutricional, la emisión de $\mathrm{CO}_{2}$ tuvo la siguiente tendencia: FM + estiércol de cerdo >FM + paja de maíz >FM (Li et al., 2013). Por lo tanto, para lograr el equilibrio de los efectos del MNI deben considerarse las características específicas de los agroecosistemas donde se aplica (Liu et al., 2014a).

\section{Conveniencia económica del manejo nutricional integrado}

Cualquier tecnología agronómica que mejoré el suelo será atractiva para los agricultores sólo si es económicamente rentable. El MNI ha demostrado esta característica. Por ejemplo, en trigo, el MNI ( $6 \mathrm{t}$ de $\mathrm{Cp} \mathrm{ha}^{-1}+34.5 \mathrm{~kg}$ de $\mathrm{N} \mathrm{ha}^{-1}$ ) mejoró la CS y generó US $\$ 8.44$ por cada US $\$ 1.00$ de inversión adicional en AOs y FMs (Demelash et al., 2014). En un estudio a largo plazo en rotaciones de sojatrigo, en los años 1 al 5 y 26 al 30 de cultivo, la relación beneficio: costo (B:C) para la soja fu mayor con MNI que con FM sola, lo que mostró que a largo plazo el MNI es más rentable, mientras que la FM no generó ganancias ni pérdidas (Bhattacharyya et al., 2008).

En arroz-trigo y algodón-trigo, el MNI mantuvo beneficios netos acumulados, B: C y retornos netos competitivos (Qazi et al., 2009) y después de 41 años de MNI, el rendimiento económico del arroz aumenta 9.34\% (Sharma et al., 2019). En diversos cultivos el MNI ha mostrado su efectividad económica, ya que elevó la productividad y rentabilidad del trigo (Singh et al., 2017) generó el mayor retorno neto, con una B: C de 1: 3.5 en el cultivo de cebolla (Allium cepa) (Jamir et al., 2013), mientras en tomate, maximizó la B: C (2.26 a 2.78) (Abedin et al., 2018). Sin embargo, en maíz, en el corto plazo (2 años), la FM al 100\% generó mayor ganancia neta que el MNI (con FM al 50\%) y la mayor B: C se dio con el control (sin FMs y sin AOs) (Hashim et al., 2015).

\section{Conclusiones}

El MNI es útil para llevar un manejo agrícola sostenible en varios ámbitos: a) mejora la calidad del suelo, agua, aire y la biodiversidad, además, ayuda a mitigar el calentamiento global, esto siempre y cuando se implemente las prácticas que permitan capturar el $\mathrm{CO}_{2}$ derivado del incremento de la actividad biológica; b) eleva la calidad de los alimentos y beneficia la salud de los consumidores; y c) mantiene rendimientos competitivos y eleva la eficiencia económica de los agroecosistemas. Múltiples estudios concuerdan en que el MNI puede disminuir la FM entre 25 a 50\%, sin afectar los rendimientos, con la mejora de la calidad de los productos agrícolas y del medio ambiente.

Sin embargo, los beneficios del MNI generalmente se dan a largo plazo y dependen de las dosis, propiedades, momentos y métodos de aplicación de los abonos orgánicos y fertilizantes minerales, así como de las interacciones con los componentes del suelo de cultivo, manejo y ambiente. Por lo tanto, deben considerarse las condiciones de cada agroecosistema y su entorno para implementar prácticas de nutrición que lleven a la sostenibilidad. 


\section{Literatura citada}

Abedin, M. S.; Hoque, M. A.; Khokon, M. A. R. and Hossain, I. 2018. Efficacy of trichocompost on the growth and yield of tomato. Univ. J. Plant Sci. 6(2):15-20.

Akter, N.; Ara, K. A.; Akand, M. H. and Alam, M. K. 2017. Vermicompost and trichocompost in combination with inorganic fertilizers increased growth, flowering and yield of gladiolus cultivar (GL-031) (Gladiolus grandiflorus L.). Adv. Res. 12(3):1-11.

Álvarez-Solís, J. D.; Gómez-Velasco, D. A.; León-Martínez, N. S. y Gutiérrez-Miceli, F. A. 2010. Manejo integrado de fertilizantes y abonos orgánicos en el cultivo de maíz. Agrociencia. 44:575-586.

Anwar, M.; Patra, D. D.; Chand, S.; Alpesh, K.; Naqvi, A. A. and Khanuja, S. P. S. 2005. Effect of organic manures and inorganic fertilizer on growth, herb and oil yield, nutrient accumulation, and oil quality of french Basil. Commun. Soil Sci. Plan. 36:1737-1746.

Bautista-Cruz, A.; Cruz-Domínguez, G. y Rodríguez-Mendoza, M. N. 2015. Efecto de bocashi y fertilizantes de liberación lenta en algunas propiedades de suelos con maíz. Rev. Mex. Cienc. Agríc. 6(1):217-222.

Bhardwaj, D.; Ansari, M. W.; Sahoo, R. K. and Tuteja, N. 2014. Biofertilizers function as key player in sustainable agriculture by improving soil fertility, plant tolerance and crop productivity. Microb. Cell Fact. 13(1):66-76.

Bhattacharyya, R.; Kundu, S.; Prakash, V. and Gupta, H. S. 2008. Sustainability under combined application of mineral and organic fertilizers in a rainfed soybean-wheat system of the Indian himalayas. Eur. J. Agron. 28:33-46.

Bünemann, E. K.; Bongiorno, G.; Bai, Z.; Creamer, R. E.; De-Deyn, G. and De-Goede, R. 2018. Soil quality-A critical review. Soil Biol. Biochem. 120:105-125.

Cai, A.; Xu, M.; Wang, B.; Zhang, W.; Liang, G.; Hou, E. and Luo, Y. 2019. Manure acts as a better fertilizer for increasing crop yields than synthetic fertilizer does by improving soil fertility. Soil Till. Res. 189:168-175.

Chandini; Kumar, R.; Kumar, R. and Prakash, O. 2019. The impact of chemical fertilizers on our environment and ecosystem. In: Sharma, P. (Ed.). Res. Trends Environ. Sci. AkiNik publications. India. 69-86 pp.

Chatterjee, R. and Khalko, S. 2013. Influence of organic amendments and inorganic fertilizers on late blight incidence and yield of tomato (Lycopersicon esculentum Mill.). Int. J. Geo. Agric. Environ. Sci. 1(1):36-38.

Chatterjee, R.; Bandyopadhyay, S. and Jana, J. C. 2014. Impact of organic amendments and inorganic fertilizers on production potential, nitrogen use efficiency and nitrogen balance in tomato (Lycopersicon esculentum Mill.). Int. J. Sci. Res. Know. 2(5):233-240.

Choudhary, M.; Panday, S. C.; Meena, V. S.; Singh, S. and Yadav, R. P. 2018. Long-term effects of organic manure and inorganic fertilization on sustainability and chemical soil quality indicators of soybean-wheat cropping system in the Indian mid-Himalayas. Agr. Ecosyst. Environ. 257:38-44.

Das, S.; Teja, K. C.; Duary, B.; Agrawal, P. K. and Bhattacharya, S. S. 2015. Impact of nutrient management, soil type and location on the accumulation of capsaicin in Capsicum chinense (Jacq.): one of the hottest chili in the world. Sci. Hortic-Amsterdam. 213:354-366.

Demelash, N.; Bayu, W.; Tesfaye, S.; Ziadat, F.; and Sommer, R. 2014. Current and residual effects of compost and inorganic fertilizer on wheat and soil chemical properties. Nutr. Cycl. Agroecosyst. 100:357-367. 
Doan, T. T.; Henry-des-Tureaux, T.; Rumpel, C.; Janeau, J. and Juoquet, P. 2015. Impact of compost, vermicompost and biochar on soil fertility, maize yield and soil erosion in northern Vietnam: a three year mesocosm experiment. Sci. Total Environ. 514(1):147-154.

Efthimiadou, A.; Bilalis, D.; Karkanis, A. and Froud-Williams, B. 2010. Combined organic/ inorganic fertilization enhance soil quality and increased yield, photosynthesis and sustainability of sweet maize crop. Australian ALCS. 4(9):722-729.

El-Naggar, A.; Lee, S. S.; Rinklebe, J.; Farooq, M. and Song, H. 2019. Biochar application to low fertility soils: A review of current status, and future prospects. Geoderma. 337:536-554.

Etchevers, J. D.; Hidalgo; Pajares, C. S.; Gallardo, J. F.; Vergara, M. Á.; Bautista, M. A. y Padilla J. 2009. Calidad o salud del suelo: conceptos, indicadores y aplicación en agricultura. (Ed.). 107-121 pp.

FAO. 1998. Organización de las naciones unidas para la agricultura y la alimentación. Guide to efficient plant nutrient management. Rome, Italy. 1-18 pp.

FAO. 2015. Agricultura sostenible. http://www.fao.org/sustainable-development-goals/overview/ fao-and-post-2015/sustainable-agriculture/es/.

FAO. 2019. Estadísticas sobre seguridad alimentaria. http://www.fao.org/economic/ ess/ess-fs/es/.

Fuentes-Ponce, M.; Moreno-Espíndola, I. P.; Sánchez-Rodríguez, L. M.; Ferrara-Guerrero, M. J. and López-Ordaz, R. 2016. Dehydrogenase and mycorrhizal colonization: tools for monitoring agrosystem soil quality. Appl. Soil Ecol. 100:144-153.

García, Y.; Ramírez, W. y Sánchez, S. 2012. Indicadores de la calidad de los suelos: una nueva manera de evaluar este recurso. Pastos y Forrajes. 35(2):125-138.

García-Mendívil, H. A.; Castro-Espinoza, L.; Guzmán-Fierros, E.; Mungarro-Ibarra, C.; ArellanoGil, M.; Martínez-Carrillo, J. L. y Gutiérrez-Coronado, M. A. 2014. Aplicación de compost, a base de champiñón enriquecida con silicio, en trigo (Triticum spp.). Agrociencia. 48:691-702.

Gupta, N.; Debnath, S.; Sharma, S.; Sharma, P. and Purohit, J. 2017. Role of nutrients in controlling the plant diseases in sustainable agriculture. In: Meena, V. S.; Mishra, P. K.; Bisht, J. K. and Pattanayak, A. (Ed.). Agriculturally important nicrobes for sustainable agriculture. Springer nature. Singapore. 217-262 pp.

Gutiérrez, E. V.; Gutiérrez, M. del C. y Ortiz, C. A. 2015. Manejo integrado de nutrientes en sistemas agrícolas intensivos: revisión. Rev. Mex. Cienc. Agríc. 6(1):201-215.

Hashim, M.; Dhar, S.; Vyas, A. K.; Pramesh, V. and Kumar, B. 2015. Integrated nutrient management in maize (Zea mays)-wheat (Triticum aestivum) cropping system. Indian J. Agr. Sci. 60(3):352-359.

Hernández, O. A.; Rivera, C. H.; Díaz, E.; Ojeda, D. and Guerrero, V. 2017. Plant and livestock waste compost compared with inorganic fertilizer: nutrient contribution to soil. Terra Latinoam. 35(4):321-328.

Hernández, T.; Chocano, C.; Moreno, J. and García, C. 2014. Towards a more sustainable fertilization: Combined use of compost and inorganic fertilization for tomato cultivation. Agric. Ecosyst. Environ. 196:178-184.

Hua, K.; Wang, D.; Guo, X. and Guo Z. 2014. Carbon sequestration efficiency of organic amendments in a long-term experiment on a vertisol in Huang-Huai-Hai Plain, China. PLoS one. 9(9):e108594.

IFA. 2018. International fertilizer association. Production and international trade and agriculture services. Fertilizer outlook. 2 p.

Ilupeju, E. A. O.; Akanbi, W. B.; Olaniyi, J. O.; Lawal, B. A.; Ojo, M. A. and Akintokun, P. O. 2015. Impact of organic and inorganic fertilizers on growth, fruit yield, nutritional and lycopene contents of three varieties of tomato (Lycopersicon esculentum (L.) Mill) in Ogbomoso, Nigeria. Afr. J. Biotechnol. 14(31):2424-2433. 
Jamir, S.; Singh, V. B.; Kanaujia, S. P. and Singh, A. K. 2013. Effect of integrated nutrient management on growth; yield and quality of onion (Allium cepa L). Progr. Hort. 45:373-380.

Kaur, S.; Dheri, G. S. and Benbi, D. 2019. Effect of long-term fertilization in maize-wheat cropping system on carbon mineralization in soil. Carbon Manag. 10(6):523-532.

Lal, R. 2018. Digging deeper: a holistic perspective of factors affecting soil organic carbon sequestration in agroecosystems. Global Change Biol. 24:3285-3301.

Li, L. J.; You, M. Y.; Shi, H. A.; Ding, X. L.; Qiao, Y. F. and Han, X. Z. 2013. Soil CO 2 emissions from a cultivated Mollisol: Effects of organic amendments, soil temperature, and moisture. Eur. J. Soil Biol. 55:83-90.

Li, J.; Wu, X.; Gebremikael, M. T.; Wu, H. and Cai, D. 2018a. Response of soil organic carbon fractions, microbial community composition and carbon mineralization to high-input fertilizer practices under an intensive gricultural system. PLoS One. 13(4):e0195144.

Li, W.; Chen, H.; Cao, C.; Zhao, Z.; Qiao, Y. and Du, S. 2018b. Effects of Long-Term Fertilization on organic carbon and nitrogen dynamics in a Vertisol in eastern China. Open J. Soil. Sci. 8:99-117.

Liu, C.; Lu, M.; Cui, J.; Li, B. and Fang, C. 2014a. Effects of straw carbon input on carbon dynamics in agricultural soils: a meta-analysis. Global Change Biol. 20:1366-1381.

Liu, C. W.; Sung, Y.; Chen, B. C. and Lai, H. Y. 2014b. Effects of nitrogen fertilizers on the growth and nitrate content of Lettuce (Lactuca sativa L.). Int. J. Environ. Res. Public health. 11:4427-4440.

Liu, Z; Rong, Q.; Zhou, W. and Liang, G. 2017. Effects of inorganic and organic amendment on soil chemical properties, enzyme activities, microbial community and soil quality in yellow clayey soil. Plos One. 12(3):e0172767.

Macías, R.; Grijalva, R. L. y Robles, F. 2012. Respuesta de la aplicación de estiércol y fertilizantes sobre el rendimiento y calidad del chile jalapeño. Biotecnia. 14(3):32-38.

Masunga, R. H.; Uzokwe, V. N.; Mlay, P. D.; Odeh, I.; Singh, A.; Buchan, D. and De Neve, S. 2016. Nitrogen mineralization dynamics of different valuable organic amendments commonly used in agriculture. Appl. Soil Ecol. 101:185-193.

McKenzie, F. and Williams, J. 2015. Sustainable food production: constraints, challenges and choices by 2050. Food Secur. 7:221-233.

Mehta, C. M.; Palni, U.; Franke-Whittle, I. H. and Sharma, A. K. 2014. Compost: its role, mechanism and impact on reducing soil-borne plant diseases. Waste Manage. 34:607-622.

Mondal, T.; Datta, J. K. and Mondal, N. K. 2017. Chemical fertilizer in conjunction with biofertilizer and vermicompost induced changes in morpho-physiological and bio-chemical traits of mustard crop. J. Saudi Soc. Agric. Sci. 16(2):135-144.

Navarrete, A.; Vela, G.; López, J. y Rodríguez, M. L. 2011. Naturaleza y utilidad de los indicadores de calidad del suelo. ContactoS. 80:29-37.

Ojo, O. I.; Olajire-Ajayi, B. L.; Dada, O. V. and Wahab, O. M. 2015. Effects of fertilizers on soil's microbial growth and populations: a review. EJER. 4(7):52-61.

Omari, R. A.; Sarkodee-Addo, E.; Fujii, Y.; Oikawa, Y. and Bellingrath-Kimura, S. D. 2017. Impacts of fertilization Type on soil microbial biomass and nutrient availability in two agroecological zones of ghana. Agronomy. 7(3):55-69.

Pant, P. K. and Ram, S. 2018. Long-term manuring and fertilization effects on soil physical properties after forty-two cycles under rice-wheat system in north indian mollisols. Int. J. Curr. Microbiol. App. Sci. 7(7):232-240. 
Pariari, A. and Khan, S. 2013. Integrated nutrient management of chilli (Capsicum annuum L.) in gangetic alluvial plains. J. Crop Weed. 9(2):128-130.

Pinto, Z. V.; Morandi, M. A. B. and Bettiol, W. 2013. Induction of suppressiveness to fusarium wilt of chrysanthemum with composted sewage sludge. Trop. Plant. Pathol. 38(5):414-422.

Postma, J. and Schilder, M. T. 2015. Enhancement of soil suppressiveness against Rhizoctonia solani in sugar beet by organic amendments. Appl. Soil Ecol. 94:72-79.

Premamali, M.; Kannangara, K. and Yapa, P. 2019. Impact of composting on growth, vitamin C and calcium content of Capsicum chinense. Sustain. Agric. Res. 8(3):57-65.

Qazi, M. A.; Akram, N.; Artiola, J. F. and Tuller, M. 2009. Economic and environmental implications of solid waste compost applications to agricultural fields in punjab, pakistan. Waste manage. 29:2437-2445.

Ram, S.; Singh, V. and Sirari, P. 2016. Effects of 41 years of application of inorganic fertilizers and farm yard manure on crop yields, soil quality, and sustainable yield index under a ricewheat cropping system on mollisols of north india. commun. Soil Sci. Plan. 47(2):179-193.

Ren, T.; Wang, J.; Chen, Q.; Zhang, F. and Lu, S. 2014. The effects of manure and nitrogen fertilizer applications on soil organic carbon and nitrogen in a high-input cropping system. Plos One. 9(5):e97732.

Santiago-Lorenzo, M. R.; López-Jiménez, A.; Saucedo-Veloz, C.; Cortés-Flores, J. I.; JaénContreras, D. y Suárez-Espinosa, J. 2016. Composición nutrimental del nopal verdura producido con fertilización mineral y orgánica. Rev. Fitotec. Mex. 39(4):403-407.

Sarkadi, L. S. 2019. Effects of fertilizer on food supply. In: Orna, M.; Eggleston, V. G. and Bopp, A. F. (Ed.). Chemistry's role in food production and sustainability: past and present. ACS symposium series. American chemical society. 1314. Washington, DC. 129-145 pp.

Sarwar, G.; Schmeisky, H.; Hussain, N.; Muhammad, S.; Ibrahim, M. and Safdar, E. 2008. Improvement of soil physical and chemical properties with compost application in ricewheat cropping system. Pak. J. Bot. 40(1):275-282.

Sharma, S.; Padbhushan, R. and Kumar, U. 2019. Integrated nutrient management in rice-wheat cropping system: an evidence on sustainability in the Indian subcontinent through metaanalysis. Agronomy. 9(2):71-85.

Shi, S.; Wang, X.; Ye, Z.; Chen, W.; Li, T.; Chen, J. and Li, J. 2019. Effect of the combined application of fungal residue and chemical fertilizers on the mineralization of soil organic carbon in paddy fields. Environ. Sci Pollut. R. 26(23):23292-23304.

Šimanský, V.; Šrank, D.; Jonczak, J. and Juriga, M. 2019. Fertilization and application of different biochar types and their mutual interactions influencing changes of soil characteristics in soils of different textures. J. Ecol. Eng. 20(5):149-164.

Singh, M.; Khan, M. M. A. and Naeem, M. 2014. Effect of nitrogen on growth, nutrient assimilation, essential oil content, yield and quality attributes in Zingiber officinale rosc. J. Saudi Soc. Agric. Sci. 15(2):171-178.

Singh V.; Rana, N. S.; Kumar, R.; Vivek, and Dhyani, B. P. 2017. Integration of organic and mineral nutrient sources enhances wheat productivity, soil health and profitability in western uttar pradesh. Int. J. Curr. Microbiol. App. Sci. 6(10):3089-3097.

Smith, L. G.; Kirk, G. J. D.; Jones, P. J. and Williams, A. G. 2019. The greenhouse gas impacts of converting food production in england and wales to organic methods. Nat. Commun. 10(4641):1-10.

Srivastava, A. K. 2012. Integrated nutrient management in citrus. In: Srivastava, A. K. (Ed.). Advances in citrus nutrition. Springer. New York-London. 369-389 pp. 
Sun, J.; Zou, L.; Li, W.; Yang, J.; Wang, Y.; Xia, Q. and Peng, M. 2018. Rhizosphere soil properties and banana Fusarium wilt suppression influenced by combined chemical and organic fertilizations. Agr. Ecosyst. Environ. 254:60-68.

Thangarajan, R.; Bolan, N. S.; Tian, G.; Naidu, R. and Kunhikrishnan, A. 2013. Role of organic amendment application on greenhouse gas emission from soil. Sci. Total Environ. 465:72-96.

Tripathi, R.; Nayak, A. K.; Bhattacharyya, P.; Shukla, A. K. and Shahid, M. 2014. Soil aggregation and distribution of carbon and nitrogen in different fractions after 41years long-term fertilizer experiment in tropical rice-rice system. Geoderma. 213:280-286.

UNDESA. 2019. United nations department of economic and social affairs/population division. World population prospects: Highlights. St/Esa/Ser.A/423. 5. p.

Verma, S.; Sharma, A.; Kumar, R.; Kaur, C.; Arora, A.; Shah, R. and Nain, L. 2015. Improvement of antioxidant and defense properties of tomato (var. Pusa rohini) by application of bioaugmented compost. Saudi J. Biol. Sci. 22(3):256-264.

Wang, J. Y.; Yan, X. Y. and Gong, W. 2015. Effect of long-term fertilization on soil productivity on the north china plain. Pedosphere. 25(3):450-458.

WHO. 2018. World health organization. Ambient (outdoor) air pollution. https://www.who.int/en/news-room/fact-sheets/detail/ambient-(outdoor)-air-quality-andhealth.

Wilson, M. G. y Sasal, M. C. 2017. Aplicación de indicadores de calidad de suelo para el monitoreo agroambiental. In: Wilson M. G. (Ed.). Manual de indicadores de calidad del suelo para las ecorregiones de Argentina. Instituto Nacional de Tecnología Agropecuaria. Argentina. 23$27 \mathrm{pp}$.

Wu, W.; Shah, F.; Shah, F. and Huang, J. 2015. Rice sheath blight evaluation as affected by fertilization rate and planting density. Australas. Plant Pathol. 44(2):183-189.

Xia, L.; Lam, S. K.; Wolf, B.; Kiese, R.; Chen, D. and Butterbach-Bahl, K. 2018. Trade-offs between soil carbon sequestration and reactive nitrogen losses under straw return in global agroecosystems. Glob. Chang. Biol. 24(12):5919-5932.

Yang, H.; Zhou, J.; Feng, J.; Zhai, S.; Chen, W.; Liu, J. and Bian, X. 2019. Ditch-buried straw return: A novel tillage practice combined with tillage rotation and deep ploughing in ricewheat rotation systems. Adv. Agron. 154 :257-290.

Zhen, Z.; Liu, H.; Wang, N.; Guo, L. and Meng, J. 2014. Effects of manure compost application on soil microbial community diversity and soil microenvironments in a temperate cropland in China. Plos One. 9(10):e108555.

Zornoza, R.; Acosta, J. A.; Bastida, F.; Domínguez, S. G.; Toledo, D. M. and Faz, A. 2015. Identification of sensitive indicators to assess the interrelationship between soil quality, management practices and human health. Soil. 1(1):173-185. 